\title{
3 Using evidence in Africa
}

\author{
A framework to assess what \\ works, how and why
}

Laurenz Langer and Vanesa Weyrauch

\section{Summary}

This chapter presents an analytical framework for investigating the effectiveness of interventions aiming to support the use of evidence in policy and practice (i.e. evidence-informed decision making (EIDM)). The analytical framework draws on two existing conceptual tools to research and understand EIDM: the Science of Using Science framework (Langer et al., 2016a) and the Context Matters framework (Weyrauch et al., 2016). It aims to present an inductive analytical tool that can be adapted and applied by decision makers, researchers and knowledge brokers to explore evidence use interventions at all stages of development from conceptualisation and planning to implementation to evaluation of interventions. It does so by providing a structured approach to categorising evidence use interventions through a mechanism typology, and to categorising evidence use outcomes by applying a behaviour change lens. Contextual factors influencing evidence use are structured and organised too. Practitioners of EIDM are thus provided with a versatile conceptual device that can be applied in investigating different facets of the process of using evidence to inform decisions.

\section{Introduction}

This chapter introduces an inductive analytical framework for conceptualising evidence use interventions and investigating their potential effects. The framework draws on existing conceptual tools for researching evidence-informed decision making (EIDM) and is aimed at supporting decision makers, researchers and knowledge brokers in exploring evidence use interventions. It does not constitute a deductive or normative framework outlining what or how interventions should lead to positive impacts on decision makers' use of evidence. The framework is intended as a versatile analytical device that can be adapted and used as an iterative lens to support the conceptualisation, implementation and evaluation of evidence use interventions.

This analytical framework was used to guide the research for and analysis of the case studies in this book. The insights and lessons emerging from the case studies were used to further refine this framework, as discussed in Chapter 13.

This chapter starts by explaining why there is a need for a new analytical framework to conceptualise evidence use interventions and their potential effects. 
It then discusses existing frameworks and analytical tools. The proposed analytical framework is then presented and illustrated in detail. The chapter concludes with thoughts on the potential application and limitations of the framework.

\section{How an analytical framework can support EIDM research and practice}

There is now a considerable body of research evaluating the effectiveness of strategies promoting EIDM/EBPM. Different types of evidence use strategies have been evaluated, focusing, for example on the impact of individual evidence champions, communities of practice or structural changes in organisational management and supervision structures. This growing number of evaluations covers multiple sectors such as health care, education, social work and international development and includes different users of evidence at a practice and policy level.

The same trend can be observed at a research synthesis level with multiple reviews attempting to bring together the results of these primary evaluations to understand what works in supporting research use (e.g. Moore et al., 2011). However, individual reviews differ in their conclusions. For example, while Yoost and colleagues' (2015) meta-analysis found that a multifaceted intervention on nurses' use of evidence (e.g. educational meetings and use of a mentor) had no effect, Hines and peers' (2015) systematic review identified interactive or activity-based learning to be effective in supporting nurses' evidence use. It is thus a challenge to generalise the findings of these reviews of what works to increase research use and to assess the patterns and directions of effects in the body of evidence.

In addition, it is challenging to compare and contrast different evidence use interventions across contexts as there is no agreed-on typology to categorise such interventions. For example, the intervention of using mentoring to support decision makers' use of evidence has been described as an intervention to support relationship-building and social influence by some commentators (e.g. Jordaan et al., 2018), whereas others see it primarily as a training approach (e.g.Yoost et al., 2015). Another difficulty is that interventions to support evidence use within government are rarely reported and discussed within the wider academic debates on EIDM. This fragmented state of conceptualisation of evidence use interventions challenges a transfer of knowledge across contexts. For example, decision makers in one country might not be aware of similar EIDM interventions and approaches in another country because they are reported and framed differently.

This characteristic of a fragmented evidence base for what works to support and institutionalise EIDM prevails in Africa too. As has happened internationally, Africa has seen a range of different evidence use interventions covering a spectrum of approaches. Examples are capacity-building programmes such as the Building Capacity to Use Research Evidence Programme (BCURE), to rapid response services such as pioneered by the Africa Centre for Rapid Evidence Synthesis, to high-level inter-governmental partnerships such as the Twende Mbele initiative, and continental evidence networks such as the Africa Evidence Network. 
A particular feature of these approaches is that a comparatively large number of interventions and instruments are driven by African governments themselves. This includes for example South Africa's National Evaluation System (Goldman, 2014) and Kenya's parliamentary evidence-based policy-making caucus.

Many of these interventions in different countries hold large potential for synergies and cross-learning, but the reality is that most EIDM interventions and most cross-learning is confined to national, and often sectoral, silos (AEN, 2019). This situation is not confined to the African evidence ecosystem. We therefore propose an analytical framework to explore evidence use interventions in Africa, in order to:

1 Structure the available research and tacit knowledge on EIDM in a consistent manner;

2 Identify patterns in this overall evidence base;

3 Support cross-learning and collaboration around synergies of different interventions and approaches promoting evidence use.

The analytical framework was tested and further refined through the research and cross-learning presented within this book.

\section{Existing frameworks and analytical tools for evidence use interventions}

In order to systematically report on and review the effectiveness of strategies for evidence use, we require a detailed conceptual framework to categorise such interventions. This framework needs to be applicable to a diverse range of contexts, types of EIDM interventions and programmes so that it can guide their comparative analysis and investigation. At the highest level, there are three types of conceptual frameworks and models in EIDM: 1) supply-side frameworks and models; 2) demand-side frameworks and models, and 3) practice-informed frameworks and theories of change. Each is briefly discussed in the following subsections.

\section{Supply-side framework and models}

The first coherent academic theories about the use and systematic contribution of evidence to government policies can be traced back to the 1970s. Drawing on a decade of work on research use in the US government's fight against poverty, Carol Weiss developed a coherent theory of research use (Weiss, 1979). Various research has since refined Weiss's initial models, with the most important advancements being the development of the two-communities theory of knowledge utilisation (Caplan, 1979), the supply-and-demand model (Landry et al., 2001) and the producer-push and user-pull model (Stone, 2002), all of which are related. Essentially all three posit that researchers and policy makers are two different professional 'tribes' with their own conventions, practices and thoughtmodels. This leads to a disconnect which needs to be bridged through active interventions, akin to learning each other's languages. Landry et al. (2001) adds 
to this basic framework the notion of supplying research and demanding research to open up the two-communities model to other groups such as civil society organisations and practitioners who can either supply or demand research too.

However, the idea that there are two distinct communities that need to be bridged or in which evidence needs to be pushed from one side into action or use on the other side seems a rather linear understanding of research use that assumes a passive user ready to consume evidence. This leaves little room for the co-construction and co-creation of knowledge (Stewart et al., 2017; Dayal, 2016) and gives a simplistic view of the realities facing policy makers and practitioners - the so-called demand side.

\section{Demand-side frameworks and models}

More recent reviews of models of evidence use criticise their strong emphasis on the supply of evidence and abstract academic definitions of use (Newman et al., 2013; Langer et al., 2017). This has led to a more inclusive theory of evidence use in which users can be co-producers of knowledge and evidence rather than mere consumers (e.g. Oliver, 2012). Policy makers and practitioners are active protagonists seeking evidence to inform their practice rather than passive recipients of research, and in the process they create demand that drives evidence generation and use. Stewart and peers (2017) argue that this shift in the conceptualisation of evidence use can be traced in a shift in language too. For example, while early conceptions described evidence use as a linear process of academics producing and pushing evidence to rational policy makers who merely take up this evidence, recent conceptions of evidence use describe an organic system spanning producers and users of evidence, and intermediates, as well as a range of other factors.

The terms 'evidence system' or 'evidence ecosystem' (Goldman, 2014; Stewart et al., 2019) reflect well the shifting consensus relating to systemic models for evidence use. However, while individual attempts have been made to conceptualise and visualise the essential elements of an evidence ecosystem and their interactions (e.g. Shepherd, 2014;AEN, 2018), there is no agreed definition of what constitutes an evidence ecosystem or how it can be developed and maintained. The monitoring and evaluation (M\&E) sector in Africa has arguably moved the furthest ahead within Africa, having established national evaluation systems driving both the supply and demand for evidence. Countries such as South Africa, Uganda, Ghana, Benin and Zimbabwe have made explicit attempts to build senior managers' awareness of the importance of evidence, and so to stimulate demand. In addition, they have been actively sharing lessons across these systems to work towards scaling and institutionalisation across the continent (Goldman et al., 2018).

This recent focus on the demand side of evidence use seems justified given the relative paucity of work in EIDM explicitly focusing on decision makers (Langer et al., 2016; Newman et al., 2013). In particular, in the context of a strong public sector interest in understanding what works to make policy and practice processes more receptive to the use of evidence, it does not seem justified to apply a rigid supply-side model to conceptualise evidence use (Dayal, 2016; Langer et al., 2017). There is now an increasing range of demand-side mechanisms and 
activities proposed by public service sector organisations, such as capacity-building for civil servants, ${ }^{2}$ evidence-informed guidelines ${ }^{3}$ and policy proposals requiring an accompanying review of evidence. Further, research and practice relating to these demand-side mechanisms is supported by a range of national governments, which has led to a third theoretical frontier: developing empirical frameworks and theories of change for the practice of evidence use. ${ }^{4}$

\section{Practice-informed frameworks and theories of change}

A last set of theories and models of EIDM does not so much aim to outline overall meta-theories of how evidence can be used by policy makers. Rather, a range of scholars has started to develop more micro-level theories of change relating to how evidence can be used in particular contexts using different interventions. Examples are the linkages and exchange model (Lomas, 2000); the context, evidence, and links model (Crewe and Young, 2002); the knowledge to action framework (Graham and Tetroe, 2009); and Parkhurst's good governance of evidence model (Parkhurst, 2017).

The good governance of evidence model is of particular interest as it deepdives into the institutional structures of evidence use. The model proposes a more holistic understanding in which EIDM advocates strive for good governance of evidence rather than 'good' use by individual decision makers.

As part of a more empirically informed understanding of EIDM, scholars have also attempted to better understand contextual variables such as barriers to, and facilitators of, evidence use, either through individual case studies (e.g. Uneke et al., 2011), primary research on decision makers' perception of evidence and its use (e.g. Cronin and Sadan, 2015) or systematic reviews of such factors (Oliver et al., 2014).

This empirical work on barriers and facilitators is further complemented by a rich body of knowledge on the role of context in influencing the use of evidence (Cairney, 2016; Crewe and Young, 2002; Shaxson et al., 2015; Weyrauch et al., 2016). Paul Cairney's work in particular highlights the importance of political factors in the use of evidence. In 2016, the learning from this work on the role of context was formalised into the Context Matters framework (Weyrauch et al., 2016), which unpacks the importance and nature of context and its interaction with evidence use.

This work on context does not focus directly on interventions to support research use (which is within an organisation's control), but rather on the factors and variables that affect use. It asks which factors interventions need to be sensitive to and work towards addressing. As a result, the work informs the design of evidence use interventions and can be used to assess how an intervention interacts with different contexts that can affect the use of evidence. However, it does not assess the effectiveness and causal impact of evidence use interventions. This last contribution is provided by an existing body of work focusing on assessing what works to support evidence use.

As earlier mentioned, there have been a number of evaluations of the effectiveness of interventions aiming to support policy makers' use of evidence. 
Langer and colleagues' (2016) systematic review of reviews identified 36 existing reviews of primary evaluations of evidence use interventions, covering 129 primary evaluations. A key challenge in the synthesis of this large primary evidence base is to identify groups of homogenous interventions to understand which of them work (or don't work) to support evidence use, how and why. A framework is required to group and categorise these interventions in order to be able to aggregate their results and synthesise findings across contexts.

A range of scholars has developed frameworks and typologies of evidence use interventions, including Nutley et al. (2007) and Gough et.al. (2011). However, despite this range of existing typologies, there is no agreed overarching theory of how to categorise evidence use interventions. Further, none of the aforementioned work attempts to unpack the outcomes of research use. This means there is an absence of conceptual work on how to categorise and define different types and measures of evidence use. Aside from Carol Weiss's 1979 definition of different types of use, Dobbins and colleagues' (2009) Global EIDM index and 3ie's evidence use categorisation, little conceptual guidance is available on how to consistently define and measure evidence use.

In summary, there is a large body of conceptual and empirical research aiming to understand the practice of evidence use. This body of work has produced a range of conceptual models and frameworks, but there is no agreed analytical tool to assess evidence use interventions in practice. In order to guide the assessment of different EIDM initiatives in a range of African countries, we therefore set out to develop an analytical framework that is fit for purpose to explore these initiatives.

\section{Developing an analytical framework for comparative analysis}

To develop our analytical framework, we adapt the framework for evidence use interventions developed by Langer and colleagues (2016) and supplement this with an analytical tool to understand contextual factors shaping the impact of evidence use interventions (Weyrauch et al., 2016). More information on the methodological development of Langer and colleagues' (2016) and Weyrauch et al.'s (2016) framework can be found in the respective publications.

\section{Applying an analytical lens for evidence use strategies and outcomes: the Science of Using Science project}

Langer et al.'s (2016) framework was developed as part of the Science of Using Science project, ${ }^{5}$ a systematic review of what works to support the use of research evidence by decision makers. The framework consists of two core components: (1) a mechanism typology to structure research use strategies and activities and (2) a behaviour change typology to structure research use outcomes. It thereby contributes a structured analytical lens for categorising and analysing different applied activities to support the use of evidence and assessing whether these have been effective in changing behaviour. 
In order to group research use strategies and activities, (e.g. capacity-building in evidence use, research communication, rapid response services), Langer and colleagues focus on the underlying mechanisms of change of these activities. Mechanisms of change are defined as the processes by which evidence use might be achieved within a given strategy or activity. For example, a networking event for decision makers and researchers might support research use by developing trusted relationships and ongoing interaction and exchange between the two groups. Relationships and interactions in this example would then be the underlying mechanism of change of the activity of hosting a networking event.

Langer et al. (2016) formulated a list of six mechanisms underlying research use interventions, which is presented in Table 3.1. ${ }^{6}$ As introduced earlier, this list builds on existing work by the authors, in particular Gough et al. (2011). Building on the first conceptual foundation and taxonomies for evidence use interventions by Walter et al. (2003) and Nutley et al. (2007), Gough et al. (2011) refined an initial concise list of mechanisms as part of the Evidence Informed Policy in Education in Europe project. The Science of Using Science project then further adapted and developed these mechanisms resulting in the final six mechanisms.

The six mechanisms are structured using a numerical list and abbreviation (M1-M6) for the purpose of accessibility. This structure does not reflect a

Table 3.1 Evidence use mechanisms

\begin{tabular}{|c|c|c|}
\hline Mechanism & Description & Example of linked activity \\
\hline $\begin{array}{l}\text { Awareness } \\
\text { (M1) }\end{array}$ & $\begin{array}{l}\text { Building awareness of, and } \\
\text { positive attitudes towards, } \\
\text { EIDM. }\end{array}$ & $\begin{array}{l}\text { - Social marketing of the } \\
\text { norm to use evidence } \\
\text { - Awareness-raising campaigns }\end{array}$ \\
\hline $\begin{array}{l}\text { Agree } \\
(\mathrm{M} 2)\end{array}$ & $\begin{array}{l}\text { Building mutual understanding } \\
\text { and agreement on policy- } \\
\text { relevant questions and the } \\
\text { kind of evidence needed to } \\
\text { answer them. }\end{array}$ & $\begin{array}{l}\text { - Co-production approaches } \\
\text { between researchers and } \\
\text { government staff } \\
\text { - Steering committees }\end{array}$ \\
\hline $\begin{array}{l}\text { Access } \\
\text { (M3) }\end{array}$ & $\begin{array}{l}\text { Providing communication of, } \\
\text { and convenient access to, } \\
\text { evidence. }\end{array}$ & $\begin{array}{l}\text { - Knowledge repositories } \\
\text { - Communication campaigns } \\
\text { and strategies }\end{array}$ \\
\hline $\begin{array}{l}\text { Interact } \\
\text { (M4) }\end{array}$ & $\begin{array}{l}\text { Interaction between decision } \\
\text { makers and researchers to } \\
\text { build trusted relationships, } \\
\text { collaborate and gain exposure } \\
\text { to a different type of social } \\
\text { influence. }\end{array}$ & $\begin{array}{l}\text { - Knowledge brokers } \\
\text { - Networks and communities } \\
\text { of practice }\end{array}$ \\
\hline $\begin{array}{l}\text { Ability } \\
\text { (M5) }\end{array}$ & $\begin{array}{l}\text { Supporting decision makers in } \\
\text { developing skills in accessing } \\
\text { and making sense of evidence. }\end{array}$ & $\begin{array}{l}\text { Capacity-building (e.g. } \\
\text { workshops and formal } \\
\text { training courses) } \\
\text { - Mentoring programmes }\end{array}$ \\
\hline $\begin{array}{l}\text { Institutionalising / } \\
\text { formalising } \\
\text { (M6) }\end{array}$ & $\begin{array}{l}\text { Influencing decision-making } \\
\text { structures and processes. }\end{array}$ & $\begin{array}{l}\text { - Secondments } \\
\text { - Embedded support (e.g. } \\
\text { knowledge brokers) }\end{array}$ \\
\hline
\end{tabular}


hierarchical order of the mechanisms, and each mechanism is assumed to be of equal importance in supporting decision makers' use of research. For each mechanism, illustrative examples of corresponding evidence use activities and interventions are provided in the right-hand column.

In order to structure evidence use outcomes, Langer and colleagues conceptualised evidence use as a form of behaviour change, that is for decision makers to increase their use of research evidence requires a change in their behaviour. Examples of evidence use include decision makers introducing evidence during policy debates, accessing and interpreting diagnostic evidence when developing a policy proposal or integrating evaluation results into programme design. Using this conceptualisation of evidence use as behaviour change, Langer et al. adopt an existing framework for characterising and designing behaviour change interventions developed by Michie et al. (2011).

Based on a review of existing frameworks for conceptualising behaviour change, Michie et al. (2011) propose a 'behaviour system', which assumes behaviour change to result from the interplay of three essential conditions: capability, opportunity, and motivation, which they termed the COM-B system. Behaviour change interventions work through changing one or more of these conditions. Langer and colleagues follow this conceptualisation of behaviour change in their framework for evidence use outcomes and retain Michie et al.s definition of capability, opportunity and motivation.

Langer and colleagues' final framework then merges their six mechanisms for structuring evidence use interventions with the COM-B system for structuring behaviour change outcomes (Figure 3.1). As Figure 3.1 indicates, all evidence

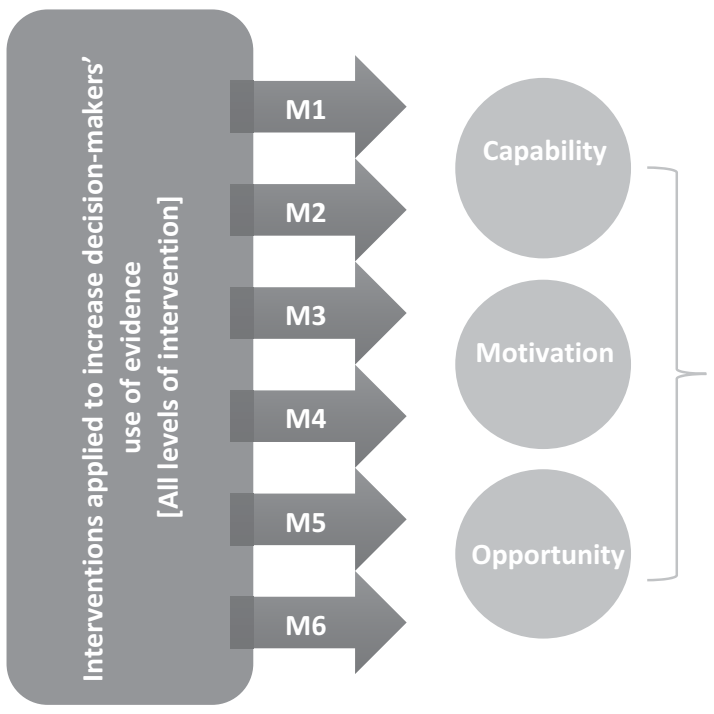

Figure 3.1 Science of Using Science conceptual framework

Source: Langer et al. (2016). 
use interventions are categorised according to the six mechanisms of change. It is these mechanisms that are assumed to be the unit of analysis in applied strategies and activities to support decision makers' use of evidence

Each of the six mechanisms (M1-M6) then works through one or more of the three COM-B components in order to effect decision makers' behaviour in relation to evidence use. This leaves the COM-B components to serve as intermediate outcomes representing the capability, opportunity and motivation to use evidence. The final outcome of evidence use is defined as a type of behaviour change of decision makers. Behaviour change in terms of evidence use may occur at different levels including individual behaviour, immediate organisational context (e.g. where people work), broader organisational context (e.g. local government) or wider national and international context.

\section{Developing an analytical lens for the context in which evidence use activities are implemented: the Context Matters framework}

Evidence use activities are not implemented in a vacuum but are highly dependent on the context of implementation. The role of context and how it shapes the use of evidence has been discussed widely in the literature on EIDM (e.g. Nutley et al., 2007; Parkhurst, 2016; Cairney, 2016), but context is often merely acknowledged as a large and general barrier or facilitator when describing the effectiveness or ineffectiveness of an intervention. In order to understand context in a more holistic and structured manner and to detect and understand the best entry points to improve the use of knowledge in a public agency, Weyrauch et al. (2016) developed an interactive and participatory tool: the Context Matters framework. This tool is a lens for understanding and acting upon internal and external factors of the context that may influence the use of evidence within an organisation.

The framework focuses specifically on the production and use of research in government institutions. Context in this tool refers to the specific environment in which people try to get research evidence and knowledge into practice. In its most simplistic form, the term includes the physical environment in which practice takes place but also encompasses the relationships and processes that go beyond this physical environment and enable change as a consequence. As with Langer and colleagues' framework for intervention and outcomes, the explicit aim of the Context Matters framework is to structure and systematise different patterns of how context influences evidence production and use in order to gain a consistent analytical lens.

Weyrauch's framework comprises six facets or 'dimensions' of context that systematically influence the use of evidence by decision makers, as presented in Table 3.2. ${ }^{7}$ These six dimensions fall into two categories: external and internal. The first two external dimensions (in grey) are (1) the macro-context and (2) intra-and inter-relationships with state and non-state agents. The four internal dimensions are (3) culture; (4) organisational capacity; (5) management and processes; and (6) core resources. Table 3.2 provides an overview of the key definitions of each of these six dimensions. 
Table 3.2 Dimensions of context according to the Context Matters framework

\begin{tabular}{|c|c|c|}
\hline $\begin{array}{l}\text { Dimension of } \\
\text { context }\end{array}$ & Description & Sub-dimensions \\
\hline 1 Macro-context & $\begin{array}{l}\text { Overarching forces at the } \\
\text { national level that establish } \\
\text { the 'bigger picture' in } \\
\text { which policy is made and, } \\
\text { consequently, how research } \\
\text { can or cannot inform it. } \\
\text { This includes the political, } \\
\text { economic, social and cultural } \\
\text { factors that surround the } \\
\text { policy-making institution and } \\
\text { in which it is embedded. }\end{array}$ & $\begin{array}{l}\text { - Usual large factors acknowledged } \\
\text { in literature (extent of political, } \\
\text { academic and media freedom, etc.) } \\
\text { - Popular pressure for change } \\
\text { - Crises and transitions } \\
\text { - Degree of power distribution in the } \\
\text { political system } \\
\text { - Prevailing policy narratives } \\
\text { - Discretional decision making and } \\
\text { corruption } \\
\text { - Strategic planning culture } \\
\text { - Consultation and participation in } \\
\text { policy processes } \\
\text { - Knowledge regime }\end{array}$ \\
\hline $\begin{array}{l}2 \text { Intra- and } \\
\text { inter- } \\
\text { institutional } \\
\text { linkages }\end{array}$ & $\begin{array}{l}\text { Refers to the relationships } \\
\text { between related government } \\
\text { agencies. Inter-institutional } \\
\text { linkages refer to an } \\
\text { agency's interaction with } \\
\text { other knowledge users } \\
\text { and producers (such as } \\
\text { universities, NGOs or think } \\
\text { tanks) which can affect or be } \\
\text { affected by policy design and } \\
\text { implementation. }\end{array}$ & $\begin{array}{l}\text { - Flow of information between } \\
\text { jurisdictions and levels } \\
\text { - Capacity to use evidence among } \\
\text { different sections and departments } \\
\text { - Support from governmental } \\
\text { agencies that produce data and } \\
\text { research } \\
\text { - Coordination among agencies } \\
\text { - Policy domains } \\
\text { - Relationships with other state } \\
\text { agencies for policy design and } \\
\text { implementation } \\
\text { - Existence and types of policy } \\
\text { forums and epistemic communities } \\
\text { - Formal channels of interaction with } \\
\text { researchers and research institutions } \\
\text { - Number and type of civil society } \\
\text { actors involved in decision } \\
\text { processes, and degree of vested } \\
\text { interests } \\
\text { - Status of consensus on the policy } \\
\text { base }\end{array}$ \\
\hline 3 Culture & $\begin{array}{l}\text { All organisations have a culture. } \\
\text { This is a set of values and } \\
\text { assumptions that are generally } \\
\text { accepted by those within the } \\
\text { organisation as 'the norm'. }\end{array}$ & $\begin{array}{l}\text { - Values and beliefs } \\
\text { - Openness to change and innovation } \\
\text { - Incentives } \\
\text { - Motivations }\end{array}$ \\
\hline $\begin{array}{l}4 \text { Organisational } \\
\text { capacity }\end{array}$ & $\begin{array}{l}\text { An organisation's ability to use its } \\
\text { resources effectively to achieve } \\
\text { its aims - in this case, to design } \\
\text { and implement public policies. } \\
\text { It includes human resources } \\
\text { and the legal framework that } \\
\text { determines how resources can } \\
\text { or cannot be used. }\end{array}$ & $\begin{array}{l}\text { - Leadership } \\
\text { - Senior management } \\
\text { - Human resources } \\
\text { - Legal capacity }\end{array}$ \\
\hline
\end{tabular}


Table 3.2 (Continued)

\begin{tabular}{|c|c|c|}
\hline $\begin{array}{l}\text { Dimension of } \\
\text { context }\end{array}$ & Description & Sub-dimensions \\
\hline $\begin{array}{l}5 \text { Management } \\
\text { and processes }\end{array}$ & $\begin{array}{l}\text { How an institution organises } \\
\text { its daily work to achieve } \\
\text { its mission and goals, from } \\
\text { planning to implementation } \\
\text { and evaluation. }\end{array}$ & $\begin{array}{l}\text { - Degree of systematic planning } \\
\text { - Existing formal processes to access } \\
\text { and use evidence in policy making } \\
\text { - Positions, including division of } \\
\text { work and roles and responsibilities } \\
\text { - Communications processes } \\
\text { - Monitoring and evaluation }\end{array}$ \\
\hline $\begin{array}{l}6 \text { Other } \\
\text { resources }\end{array}$ & $\begin{array}{l}\text { Key resources that affect how } \\
\text { an organisation systematically } \\
\text { gathers and uses evidence, } \\
\text { including its budget and } \\
\text { technology. }\end{array}$ & $\begin{array}{l}\text { - Budget committed to research } \\
\text { - Technology } \\
\text { - Existence of a knowledge } \\
\text { infrastructure } \\
\text { - Time availability }\end{array}$ \\
\hline
\end{tabular}

The links between these dimensions of context are various and changing. For example, a restrictive macro-context will limit the room for change in most of the internal context dimensions. However, Weyrauch and colleagues stress that there are a number of sub-dimensions of the six main facets that can positively shape the overall contextual environment. These sub-dimensions refer to (1) leadership of EIDM by individual decision makers; (2) organisational culture as a key determinant of effective management processes for EIDM; and (3) staff incentives and motivation to use evidence.

In sum, the Context Matters framework aims to help decision makers better assess the contexts in which they operate and, based on careful assessment, detect where the potential for change may be greater and barriers more significant. It can thereby serve as an effective analytical lens to unpack already implemented evidence use strategies as well as plan new ones in government departments and provide a coherent structure to organise and describe how context affected or may affect these activities.

\section{Developing a combined analytical framework: synergies between the Science of Using Science and Context Matters}

In a last step, we merged the Science of Using Science's framework for unpacking evidence use interventions and outcomes with the Context Matters framework to create a tool to explore variables affecting decision makers' evidence use. Figure 3.2 outlines the combined analytical framework.

The combined analytical framework explores the evidence process from generation through to potential development impact. Each section in the framework presents an independent element that can be unpacked in more detail. The framework starts with the demand for evidence, which is assumed to be a key contextual feature that affects the production of evidence, the applied evidence use intervention, its underlying change mechanism, and the intermediate changes. 


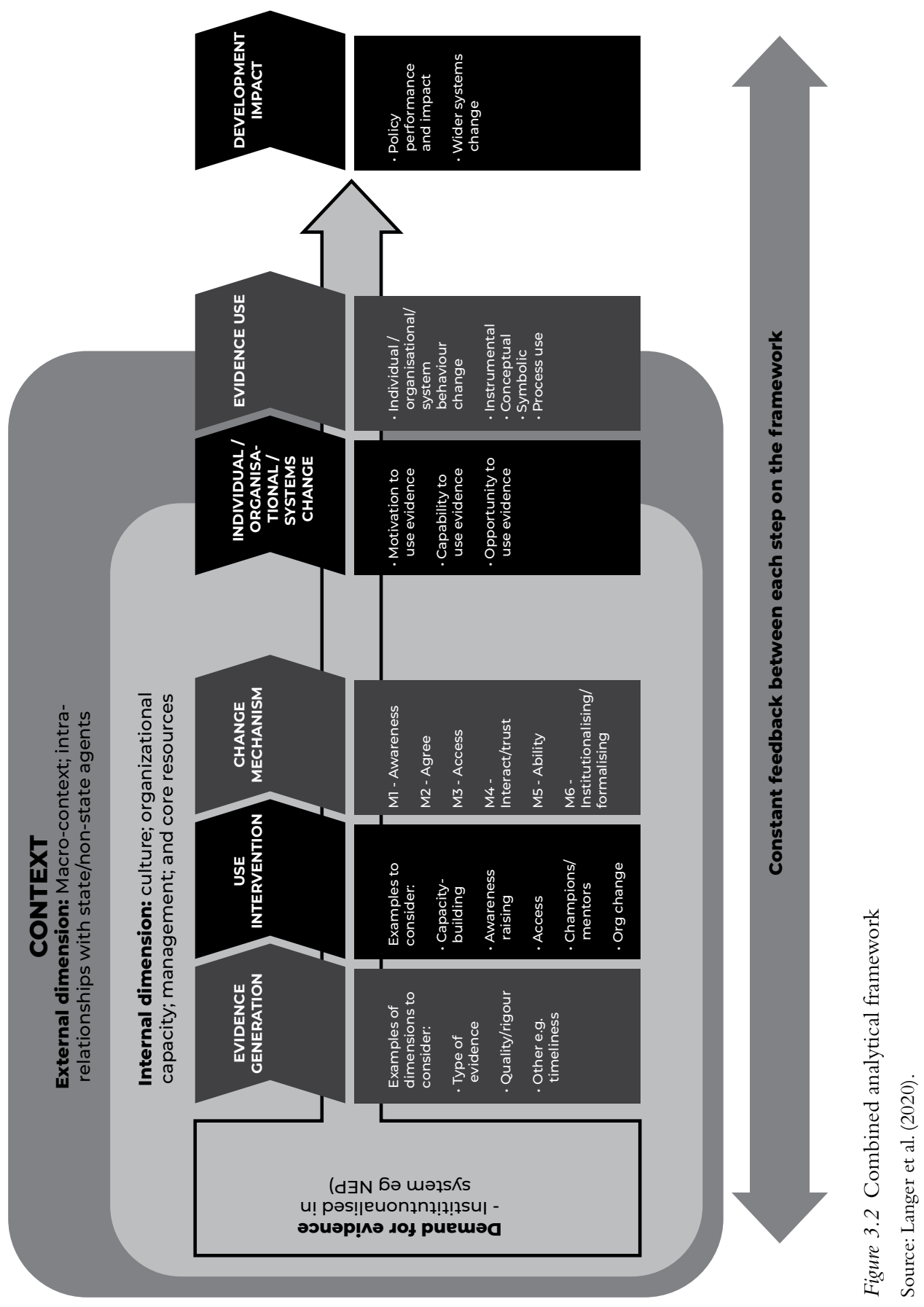


The demand for evidence refers to decision makers' and evidence users' pull for evidence. This user pull is deliberately the starting point of the framework and reflects a more government-focused rather than researcher-focused application of the framework. It suggests that efforts to increase the use of evidence ideally start with an in-depth understanding of the demand for such evidence and its existing use. The framework is thus firmly demand-led.

The second section of the framework refers to the production or supply of evidence (evidence generation). There is a range of factors to be considered when assessing the nature of evidence generation. Three key aspects related to the supply of evidence include quality, type and evidence claim. First, the quality of the evidence is likely to strongly affect its use. We would caution against a narrow interpretation of quality as methodological rigour and instead refer to quality and relevance to a policy context (Parkhurst, 2016). Second, the type of evidence matters; different types of evidence answer different questions and thus can inform different policy decisions. Third, the specific evidence claim and the standard of evidence needs to be assessed. Policy decisions should be informed by strong bodies of evidence that support specific evidence claims and comply with explicit standards of evidence set by decision-making bodies (Gough and White, 2018).

The third section of the framework investigates evidence use interventions. This refers to any programme, instrument, strategy or activity that is a deliberate and tangible input to support the use of evidence. The term 'intervention' indicates that a deliberate and tangible effort is made to intervene in the status quo in order to effect change in relation to evidence use. As outlined earlier, there is a plethora of evidence use interventions, and the Science of Using Science review alone identified 121 . The analytical framework only provides a few selected examples of such interventions.

Given the diversity and complexity of evidence use interventions, section 4 of the framework suggests that these work through six underlying mechanisms of change (M1-M6). This assumes that one can understand a given intervention through a change mechanism lens rather than investigating the interventions as a whole. For example, an EIDM mentoring programme would be assessed by unpacking its relevant mechanisms of change, which could include: ability (M5) as mentors might support a technical skill such as critical appraisal capacity; interaction (M4) as mentors and mentees assumingly connect and build a trusted relationship; and access (M3) as mentors might support mentees in accessing academic databases or linking them to sources of evidence. Most evidence use interventions are likely to employ a range of mechanisms, and it is often the precise interplay of different mechanisms that unlocks change.

Section 5 of the framework then moves from the intervention side to the outcome side, that is the use of evidence by decision makers. It assumes that intervention outcomes can be observed at an individual, organisational and systems level. For instance, the mentoring example discussed could support either an individual decision maker or the organisation that she works for, or both, depending on the content of the mentoring as well as the seniority of the mentee. This immediate or intermediate outcome of the evidence use intervention 
can be broken down into supporting capability to use evidence, motivation to use evidence, and opportunity to use evidence. To illustrate these, examples of these immediate outcomes are provided in Table 3.3.

Keeping to the EIDM mentoring example, we would then investigate how different mechanisms of change (e.g. ability, interact, access) have affected the three intermediate outcomes of capability, motivation, and opportunity to use evidence. For example, interaction with an EIDM mentor might motivate a mentee to pursue evidence by decreasing the person's isolation and providing encouragement and support. Likewise, the mentoring might provide the individual with technical EIDM skills, such as appraising a piece of evidence.

Section 6 of the framework then explores whether these intermediate outcomes did translate into actual use of evidence. It is important to note that the framework does not prescribe what constitutes a meaningful or 'positive' use of evidence. For example the sought-after instrumental use of evidence that directly leads to a more evidence-informed decision or action is but one (and rare) form of use.

In the last step (section 7), the framework encourages an investigation into whether the actual use of evidence did translate into changes in policy performance or wider systems change. ${ }^{8}$ There is no guarantee that an evidence-informed policy or programme leads to better socio-economic outcomes or that an organisational structure receptive to the use of evidence leads to improved organisational performance. Similarly, some interventions might have the power to effect systemic change across decision-making sectors (e.g. the National Evaluation System in South Africa). However, caution must be applied when investigating this last step on the framework.

The context in which this process takes place - from evidence generation to designing an evidence intervention, effecting change related to evidence use and policy impact, is indicated by the two overarching boxes that embed the

Table 3.3 Immediate outcomes

\begin{tabular}{|c|c|c|c|}
\hline Immediate outcome & Individual change & Organisational change & Systems change \\
\hline $\begin{array}{l}\text { Capability to use } \\
\text { evidence }\end{array}$ & $\begin{array}{l}\text { Skills to search } \\
\text { for evidence }\end{array}$ & - Access to databases & $\begin{array}{l}\text { Diverse supply of } \\
\text { training and capacity } \\
\text { support around } \\
\text { evidence use }\end{array}$ \\
\hline $\begin{array}{l}\text { Opportunity to } \\
\text { use evidence }\end{array}$ & $\begin{array}{l}\text { - Timely access } \\
\text { to relevant } \\
\text { evidence }\end{array}$ & $\begin{array}{l}\text { - Organisational } \\
\text { processes that create } \\
\text { a space for evidence } \\
\text { (e.g. submission } \\
\text { standards, } \\
\text { performance } \\
\text { appraisal) }\end{array}$ & $\begin{array}{l}\text { - Investment in tailored } \\
\text { evidence systems }\end{array}$ \\
\hline $\begin{array}{l}\text { Motivation to use } \\
\text { evidence }\end{array}$ & $\begin{array}{l}\text { - Awareness of } \\
\text { the value of } \\
\text { evidence use }\end{array}$ & $\begin{array}{l}\text { Organisational } \\
\text { norms support the } \\
\text { use of evidence }\end{array}$ & $\begin{array}{l}\text { Investment in diverse } \\
\text { evidence networks } \\
\text { advocating for the use } \\
\text { of evidence }\end{array}$ \\
\hline
\end{tabular}


EIDM process. As per the Context Matters framework, the context is divided into an external dimension and an internal dimension. The external dimension is indicated in a darker shade and comprises (1) the macro-context and (2) intraand inter-relationships with state and non-state agents. Both these variables are external to an evidence use intervention and cannot be significantly affected by it; they depend on larger forces and a myriad of external actors. However, certain evidence use interventions and mechanisms could alter external contexts, for example, by forging strategic alliances and networks between different actors in the evidence ecosystem. An illustration of this is how the Ghanaian Environmental Protection Agency decided to act upon opportunities to collaborate with a wider range of stakeholders, including promoting more citizen engagement to revive multi-stakeholder networks (INASP, 2018).

The four internal dimensions of context (lighter shade) can be more directly affected by an evidence use intervention. These four dimensions are (3) culture, (4) organisational capacity, (5) management and processes and (6) core resources. These internal dimensions only extend across the first five sections of the framework until the intermediate outcomes, while the external dimensions extend until the sixth section, which is the final outcome of evidence use. This differentiation aims to capture that the internal dimensions of context usually are changed through the intervention itself. For example, an intervention might actively build evidence champions to change the organisational culture around evidence use.

Yet both dimensions of context have a bearing on the demand for evidence, its generation, the identification of relevant intervention approaches and change mechanisms as well as the intermediate evidence use outcomes. While both dimensions of context affect the demand for evidence, demand for evidence is primarily driven by internal context dimensions such as organisational culture and capacity; for this reason, the demand for evidence box is shaded similarly to the internal context dimensions.

Last, none of the context boxes extend to the development impact itself, as it is assumed that a different set of contexts, not linked to the EIDM intervention itself, affects these outcomes, which relate to the particular sector itself. For example, while macro-contexts such as the prevailing political climate affect the nature of the EIDM process, a completely different set of macro-factors determines whether a particular sectoral policy has positive socio-economic impacts (e.g. economic growth, social cohesion), and these factors are not linked to the EIDM process at all.

The framework acknowledges that in practice the flow of the suggested sections is not linear. There are multiple feedback loops between evidence generation and interventions and between intermediate outcomes and demand for evidence, and between all spheres of the context and the facets of the evidence use interventions and outcomes. The arrow at the bottom of the diagram serves as a placeholder to visualise these multiple interactions and feedback loops between all parts of the framework. 


\section{Intended application and limitations of the analytical framework}

The intent of the analytical framework is to provide a consistent conceptual lens to analyse what happened in the diverse case studies of EIDM in Africa that have been collected in this volume. The consistent use of the framework as a guide and lens for analysis allows for the structured identification of transferable lessons learned and facilitates a synthesis of the insights and knowledge generated by the diverse case studies.

It is crucial to stress that the framework does not aim to reflect or prescribe how evidence use should take place; ${ }^{9}$ neither does it prescribe how evidence use interventions should be analysed. It is intended as an analytical lens with different conceptual devices, which EIDM practitioners and scholars can apply as best fits their context. In some circumstances, the COM evidence use structure might help an analysis, whereas in others, it might be the dimensions of context; each conceptual device of the framework can be applied in its own right. The framework can therefore best be regarded as a starting point to guide EIDM research and practice and not does not constitute a static blueprint or one-sizefits-all device. We hope that EIDM scholars and practitioners will apply the framework in this inductive spirit and adapt its structure and application.

This analytical framework naturally has a number of limitations. First, it is not meant to describe or capture a holistic evidence ecosystem. It does not reflect all of the actors involved in evidence production and use and how they interact; neither does it facilitate sectoral assessments. Second, the framework is not well suited to inform diagnostic studies on EIDM such as perceptions of evidence use. It is designed for, and most useful for, thinking through and planning, implementing and evaluating tangible evidence use interventions. As a result, it best fits contexts where there is an existing practice of EIDM and an intention to promote evidence use, or at least a familiarity with the notion of evidence use. Third, the framework is not focused on the processes of evidence production. It approaches EIDM from a policy-maker and practitioner perspective with a strong focus on the demand side. As a result, it is most applicable to evidence users aiming to systematise the practice of EIDM, organisations that have an interest in strengthening their use of evidence, and organisations and teams working towards supporting decision makers in their use of evidence. Fourth, as with all analytical frameworks, the conceptual and analytical devices are necessarily aggregated and abstracted. This comes at the expense of detail relating to certain concepts. For example, the framework does not provide sub-frameworks and analytical tools for specific interventions such as EIDM capacity-building, even though these are widely available. Likewise, Michie et al.'s original COM-B framework goes into more detail on how to support behaviour change. Users of the framework are encouraged to complement it with more granular analytical tools for specific evidence use interventions, mechanisms and outcomes where relevant. Fifth, and last, the 
framework is only partially empirically informed. Both the Context Matters framework and the Science of Using Science framework have been developed based on decision makers' feedback and experiences and have been applied and adapted in an EIDM practice context since their publication. However, this combined analytical framework has not been applied in practice before and is open to adaption following the experience of the case study authors using it. Some proposed changes are included in Chapter 13.

\section{Conclusion}

This chapter presents an analytical framework for investigating the effectiveness of interventions aiming to support the use of evidence in policy and practice. The analytical framework draws on two existing conceptual tools to research and understand EIDM: the Science of Using Science framework (Langer et al., 2016) and the Context Matters framework (Weyrauch et al., 2016) and has been developed through a dialogue between the authors of the two frameworks and the researchers involved in this book, many of whom come from a policy background. It aims to present an inductive analytical tool that can be adapted and applied by decision makers, researchers, and knowledge brokers to explore evidence use interventions at all stages of development: from conceptualisation and planning to implementation and evaluation of interventions.

\section{Notes}

1 Laurenz Langer developed the manuscript for this chapter. Laurenz is a co-author of the Science of Using Science report, which was part of a wider project led by David Gough and Janice Tripney from the UCL EPPI-Center and who share intellectual ownership of the Science of Using Science framework applied in this chapter. Vanesa Weyrauch read, reviewed, and commented on the chapter. She is the lead investigator of the Context Matters framework, jointly developed by INASP and Politics\&Ideas. The co-editors Ian Goldman and Mine Pabari have supported the development of the combined conceptual framework.

2 www.mandelaschool.uct.ac.za/gsdpp/courses/evidence_based_policy_making_imple mentation

3 www.afidep.org/index.html

4 www.dpme.gov.za/keyfocusareas/Socio\%20Economic\%20Impact $\% 20$ Assessment $\% 20$ System/Pages/default.aspx

5 https://eppi.ioe.ac.uk/cms/Default.aspx?tabid=3504

6 Note that there are two semantic changes in Table 3.1. In Langer et al.'s initial framework work, M3 was labelled as 'Communication \& access' and M5 as 'Skills'. Following stakeholder feedback, we have adapted the labels of these two mechanisms for the purpose of this research project.

7 With an interactive version of the framework provided online: http://cm.politicsandideas. org/homepage-old.

8 It should also be noted that evidence use does not necessarily lead to any policy changes. An evidence-based decision could be to do nothing and remain with the status quo. 
9 While applicable to all sections of the framework, this non-normative framing applies strongly to the last impact section of the framework as few individual evidence use interventions will be able to target and achieve impact at this scale.

\section{References}

Africa Evidence Network. 2018. African evidence ecosystem maps. Retrieved from https://aenwebsite.azurewebsites.net/en/learning-space/

Africa Evidence Network. 2019. A geo-map of EIDM organization in Africa. Retrieved from www.google.com/maps/d/u/0/viewer?mid=1j7yA9hfXnUio7WaRA2Ha2f4Ihr4Knng P\&ll $=7.062733884621437 \% 2 \mathrm{C} 14.946899331250052 \& z=3$

Cairney, P. 2016. The politics of evidence-based policy making. New York: Springer.

Caplan, N. 1979. The two-communities theory and knowledge utilization. The American Behavioral Scientist, 22(3), 459-471.

Crewe, E. and Young, M.J. 2002. Bridging research and policy: Context, evidence and links. London: Overseas Development Institute.

Cronin, G. and Sadan, M. 2015. Use of evidence in policy making in South Africa: An exploratory study of attitudes of senior government officials. African Evaluation Journal, 3(1), 10-20.

Dayal, H. 2016. Using evidence to reflect on South Africa's 20 years of democracy - Insights from within the policy space. Knowledge Sector Initiative Working Paper 7.

Dobbins, M., Hanna, S.E., Ciliska, D., Manske, S., Cameron, R., Mercer, S.L., O’Mara, L., DeCorby, K. and Robeson, P. 2009. A randomized controlled trial evaluating the impact of knowledge translation and exchange strategies. Implementation Science, 4(1), 61.

Goldman, I. 2014. Using evidence by government in South Africa. Retrieved from www.africaevi dencenetwork.org/wp-content/uploads/2014/12/Using-Evidence-by-Government-inSouth-Africa.pdf

Goldman, I., et al. 2018. The emergence of government evaluation systems in Africa: The case of Benin, Uganda and South Africa. African Evaluation Journal, 6(1), 1-11.

Gough, D., Tripney, J., Kenny, C. and Buk-Berge, E. 2011. Evidence informed policy in education in Europe: EIPEE final project report. London: EPPI-Centre, Social Science Research Unit, Institute of Education, University of London.

Gough, D. and White, H. 2018. Evidence standards and evidence claims in web based research portals. London: Centre for Homelessness Impact.

Graham, I.D. and Tetroe, J.M. 2009. Getting evidence into policy and practice: Perspective of a health research funder. Journal of Canadian Academy Child Adolescence Psychiatry, 18, $46-50$.

Hines, S., Ramsbotham, J. and Coyer, F. 2015. The effectiveness of interventions for improving the research literacy of nurses: A systematic review. Worldviews on Evidence-Based Nursing, 12(5), 265-272.

INASP. 2018. Context Matters Framework case study: Supporting organizational change to improve the use of evidence in environmental protection in Ghana. Retrieved from www.inasp.info/ publications/context-matters-ghana

Jordaan, S., et al. 2018. Reflections on mentoring experiences for evidence-informed decision-making in South Africa and Malawi. Development in Practice, 28(4), 456-467.

Landry, R., Amara, N. and Lamari, M. 2001. Utilization of social science research knowledge in Canada. Research Policy, 30(2), 333-349. 
Langer, L., Erasmus, Y., Tannous, N. and Stewart, R. 2017. How stakeholder engagement has led us to reconsider definitions of rigour in systematic reviews. Environmental Evidence, 6(20).

Langer, L., Tripney, J. and Gough, D. 2016. The science of using science. Researching the use of research evidence in decision-making. Technical Report. London: EPPI-Center, Social Science Research Unit, UCL Institute of Education.

Langer, L., Goldman, I. and Pabari, M. 2020. Analytical framework used to guide case study research. In Using evidence for policy and practice—Lessons from Africa. Routledge, Taylor \& Francis Group.

Lomas, J. 2000. Using linkage and exchange to move research into policy at a Canadian Foundation. Health Affairs, 19(3), 236.

Michie, S., van Stralen, M.M. and West, R. 2011. The behaviour change wheel: A new method for characterising and designing behaviour change interventions. Implementation Science, 6(1), 42.

Moore, G., Redman, S., Haines, M. and Todd, A. 2011. What works to increase the use of research in population health policy and programmes: A review. Evidence E Policy: A Journal of Research, Debate and Practice, 7(3), 277-305.

Newman, K., Capillo, A., Famurewa, A., Nath, C. and Siyanbola, W. 2013. What is the evidence on evidence-informed policy making? Lessons from the International Conference on Evidence-Informed Policy Making. Oxford: INASP.

Nutley, S.M., Walter, I. and Davies, H.T. 2007. Using evidence: How research can inform public services. Bristol, UK: Policy Press.

Oliver, K., Innvar, S., Lorenc, T., Woodman, J. and Thomas, J. 2014. A systematic review of barriers to and facilitators of the use of evidence by policymakers. BMC Health Services Research, 14(1), 1.

Oliver, S. 2012. Making a difference with systematic reviews. In Gough, D., Oliver, S. and Thomas, J. (eds.), An introduction to systematic reviews. London: Sage.

Parkhurst, J.O. 2017. The politics of evidence: From evidence-based policy to the good governance of evidence. Abingdon: Routledge.

Shaxon et al. 2015. Evidence-informed policymaking in practice: an overview from South Africa's Department of Environmental Affairs. Republic of South Africa: Department of Environmental Affairs.

Shepherd, J. 2014 How to achieve more effective services: The evidence ecosystem. Cardiff: What Works Network.

Stewart, R., Dayal, H. and Langer, L. 2017. Terminology and tensions within evidenceinformed decision-making in South Africa over a 15-year period. Research for All, 1(2), $252-264$.

Stewart, R., Dayal, H., Langer, L. and van Rooyen, C. 2019. The evidence ecosystem in South Africa: Growing resilience and institutionalisation of evidence use. Palgrave Communications, 5(1), 1-12.

Stone, D. 2002. Using knowledge: The dilemmas of 'bridging research and policy. Compare, 32(3), 285-296.

Uneke, C.J., Ezeoha, A.E., Ndukwe, C.D., Oyibo, P.G., Onwe, F., Igbinedion, E.B. and Chukwu, P.N. 2011. Individual and organisational capacity for evidence use in policy making in Nigeria: An exploratory study of the perceptions of Nigeria health policy makers. Evidence \& Policy, 7(3), 251-276.

Walter, I., Nutley, S. and Davies, H. 2003. Developing a taxonomy of interventions used to increase the impacts of research. Research Unit for Research Utilisation, ESRC Network for Evidence Based Policy and Practice. 
Weiss, C.H. 1979. The many meanings of research utilization. Public Administration Review, 39(5), 426-431.

Weyrauch, V., Echt, L. and Suliman, S. 2016. Knowledge into policy: Going beyond context matters. Retrieved from www.politicsandideas.org/wp-content/uploads/2016/07/Goingbeyond-context-matters-Framework_PI.compressed.pdf

Yost, J., et al. 2015. The effectiveness of knowledge translation interventions for promoting evidence-informed decision-making among nurses in tertiary care: A systematic review and meta-analysis. Implementation Science, 10(1), 98. 